\title{
Declining responsiveness of childhood Plasmodium falciparum infections to artemisinin-based combination treatments ten years following deployment as first-line antimalarials in Nigeria
}

Akintunde Sowunmi 1,2,3,4*, Godwin Ntadom ${ }^{1,2}$, Kazeem Akano 1,2,5, Folasade O. Ibironke ${ }^{4}$, Adejumoke I. Ayede ${ }^{6}$, Chimere Agomo ${ }^{1,7}$, Onikepe A. Folarin ${ }^{5}$, Grace O. Gbotosho ${ }^{2,3,8}$, Christian Happi ${ }^{1,5}$, Stephen Oguche ${ }^{1,9}$, Henrietta U. Okafor ${ }^{1,10}$, Martin Meremikwu ${ }^{1,11}$, Philip Agomo 1,12, William Ogala ${ }^{1,13}$, Ismaila Watila,14, Olugbenga Mokuolu ${ }^{1,15}$, Finomo Finomo ${ }^{1,16}$, Joy C. Ebenebe ${ }^{1,17}$, Nma Jiya ${ }^{1,18}$, Jose Ambe ${ }^{1,19}$, Robinson Wammanda ${ }^{1,13}$, George Emechebe ${ }^{1,20}$, Wellington Oyibo ${ }^{1,21}$, Francis Useh ${ }^{1,22}$, Temitope Aderoyeje ${ }^{4}$, Titilope M. Dokunmu², Omobolaji T. Alebiosu², Sikiru Amoo², Oluwabunmi K. Basorun², Olubunmi A. Wewe'2, Chukwuebuka Okafor ${ }^{2}$, Odafe Akpoborie ${ }^{2}$, Bayo Fatunmbi ${ }^{1,24}$, Elsie O. Adewoye ${ }^{25}$, Nnenna M. Ezeigwe ${ }^{1}$ and Ayoade Oduola ${ }^{26}$

\section{Abstract}

Background: The development and spread of artemisinin-resistant Plasmodium falciparum malaria in Greater Mekong Subregion has created impetus for continuing global monitoring of efficacy of artemisinin-based combination therapies (ACTs). This post analyses is aimed to evaluate changes in early treatment response markers 10 years after the adoption of ACTs as first-line treatments of uncomplicated falciparum malaria in Nigeria.

Methods: At 14 sentinel sites in six geographical areas of Nigeria, we evaluated treatment responses in 1341 children under 5 years and in additional 360 children under 16 years with uncomplicated malaria enrolled in randomized trials of artemether-lumefantrine versus artesunate-amodiaquine at 5-year interval in 2009-2010 and 2014-2015 and at 2-year interval in 2009-2010 and 2012-2015, respectively after deployment in 2005.

\footnotetext{
*Correspondence: akinsowunmi@hotmail.com

${ }^{1}$ Antimalarial Therapeutic Efficacy Monitoring Group, National Malaria Elimination Programme, The Federal Ministry of Health, Abuja, Nigeria ${ }^{2}$ Department of Pharmacology and Therapeutics, University of Ibadan, Ibadan, Nigeria

Full list of author information is available at the end of the article
}

(c) The Author(s). 2019 Open Access This article is distributed under the terms of the Creative Commons Attribution 4.0 International License (http://creativecommons.org/licenses/by/4.0/), which permits unrestricted use, distribution, and reproduction in any medium, provided you give appropriate credit to the original author(s) and the source, provide a link to the Creative Commons license, and indicate if changes were made. The Creative Commons Public Domain Dedication waiver (http://creativecommons.org/publicdomain/zero/1.0/) applies to the data made available in this article, unless otherwise stated. 
(Continued from previous page)

Results: Asexual parasite positivity 1 day after treatment initiation (APPD1) rose from 54 to $62 \%$ and 2 days after treatment initiation from 5 to 26\% in 2009-2010 to 2014-2015 ( $P=0.002$ and $P<0.0001$, respectively). Parasite clearance time increased significantly from 1.6 days (95\% confidence interval [Cl]: 1.55-1.64) to 1.9 days (95\% Cl, 1.9-2.0) and geometric mean parasite reduction ratio 2 days after treatment initiation decreased significantly from 11000 to 4700 within the same time period $\left(P<0.0001\right.$ for each). Enrolment parasitaemia $>75000 \mu \mathrm{l}^{-1}$, haematocrit $>27 \% 1$ day post-treatment initiation, treatment with artemether-lumefantrine and enrolment in 2014-2015 independently predicted APPD1. In parallel, KaplanMeier estimated risk of recurrent infections by day 28 rose from 8 to 14\% (P=0.005) and from 9 to 15\% $(P=0.02)$ with artemether-lumefantrine and artesunate-amodiaquine, respectively. Mean asexual parasitaemia half-life increased significantly from $1.1 \mathrm{~h}$ to $1.3 \mathrm{~h}$ within 2 years $(P<0.0001)$.

Conclusions: These data indicate declining parasitological responses through time to the two ACTs may be due to emergence of parasites with reduced susceptibility or decrease in immunity to the infections in these children.

Trial registration: Pan African Clinical Trial Registration PACTR201508001188143, 3 July 2015; PACTR201508001191898, 7 July 2015 and PACTR201508001193368, 8 July 2015 PACTR201510001189370, 3 July 2015; PACTR201709002064150, 1 March 2017; https://www.pactr.samrca.ac.za

Keywords: Declining responsiveness, Falciparum malaria, Children, Artemisinin-based combination treatment, Nigeria

\section{Multilingual abstracts}

Please see Additional file 1 for translations of the abstract into the five official working languages of the United Nations.

\section{Background}

The emergence and spread of resistance in Plasmodium falciparum to artemisinin in the Greater Mekong Subregion (GMS) [1-7] threatens the treatment and control of $P$. falciparum malaria globally [8]. Although in Africa, there is currently no evidence of resistance in $P$. falciparum to artemisinin in indigenous population [9-11], declining responsiveness manifested as increasing proportion of patients with residual asexual parasitaemia (asexual parasite positivity) 1 day after treatment initiation (APPD1) and apparent increases in rate of recrudescent infections following artemisinin-based combination therapies (ACTs) has been reported from an area of seasonal intense transmission on the coast of Kenya [12].

One of the measures of declining efficacy to artemisinin-like drugs is a terminal elimination half-time of parasitaemia $\geq 5 \mathrm{~h}$ [8]. In Africa where transmission is high and the burden of malaria is greatest, there is scant data on terminal elimination half-time of parasitaemia following ACTs [9]. In addition, most reported studies on the estimation of terminal elimination half-time of asexual parasitaemia have employed parasite clearance estimator $[8,9]$ instead of kinetic models which required intense blood sampling.

It is over a decade many African countries adopted and deployed ACTs as first-line treatments of uncomplicated falciparum malaria [13]. In Nigeria, ACTs were adopted and deployed in 2005 [14]. Despite a relatively long period of adoption and deployment, there are few reported periodic country-wide evaluations of the efficacy of ACTs on the African continent [11]. Using data from two country-wide, open-label, randomized efficacy trials at 14 sentinel sites located in six geographical areas of Nigeria over a 5-year interval (2009-2010 and 2014-2015), and at one of the sentinel sites over a 2-year interval (2009-2010 and 2012-2015) [15, $16]$, we performed a post hoc analyses over time of the in vivo responses to two ACTs, namely: artemether-lumefantrine (AL) and artesunate-amodiaquine (AA) to determine if there was declining responsiveness of childhood $P$. falciparum infections over time following adoption and deployment of the two ACTs as first-line therapies. The studies coincided with five and ten years of almost exclusive firstline treatments with ACTs for all cases of uncomplicated falciparum malaria in Nigeria.

The main objectives of our post hoc analyses are: (1) to determine if there are significant differences in early response markers (for examples, APPD1, residual asexual parasitaemia [asexual parasite positivity] 2 days after treatment initiation [APPD2], parasite reduction ratio 1 or 2 days after treatment initiation [PRRD1 or PRRD2]) and the factors contributing to these significant differences during a five-year study interval; (2) to estimate the terminal elimination half-time of asexual parasitaemia in a subpopulation of children after initiation of ACTs after a 2 year interval of almost exclusive use of ACTs and (3) to determine if there are significant differences in the terminal elimination half-time of asexual parasitaemia following a short period of use of ACTs at one of the sentinel sites.

\section{Methods}

\section{Study locations}

The initial studies were conducted between 2009 and 2010 (the end of the first 5 years of deployment) and 
between 2014 and 2015 (the end of the second 5 years of deployment). They were nested in a National Malaria Elimination Programme for monitoring the therapeutic efficacies of antimalarial drugs at 14 sentinel sites located in six geographical areas of Nigeria, namely: Agbani, Ikot Ansa, Barkin Ladi, Damboa, Ijede, Sabo quarters of Ibadan and Makarfi in Enugu, Cross River, Plateau, Borno, Lagos, Oyo, and Kaduna States, respectively in 2009-2010; and in Ogbia (Otuasegha), Neni, Ogwa, Numan, Ilorin, Kura, Bodinga and Ibadan in Bayelsa, Anambra, Imo, Adamawa, Kwara, Kano, Sokoto and Oyo States, respectively in 2014-2015 (Fig. 1). At virtually all of the sentinel sites, malaria transmission occurs all year round; however, it is more intense during the rainy season from April to October.

\section{Design of post hoc analyses}

This is a detailed evaluation of treatment responses at two time periods in open label, randomized trials conducted to monitor the efficacies of AL and AA in under 5-year-old Nigerian children with acute, symptomatic apparently uncomplicated falciparum malaria (Clinical Trial Registration Numbers PACTR201510001189370 and PACTR201709002064150). The primary efficacy endpoints were complete clearance of initial asexual parasitaemia and 28-day cure rate adjusted for re-infection by polymerase chain reaction (PCR) in 2009-2010 [15] but extended to 42 days in 2014-2015 [16]. The secondary endpoints were residual asexual parasitaemia one, two and three days after treatment initiation, parasite reduction ratios one or two days after treatment initiation, fever clearance, and gametocyte carriage. Assuming a cure rate of 100 and $95 \%$ with AL and AA, respectively, and a 5\% drop out rate, we estimated a minimum of 50 patients per treatment arm in each sentinel site would provide 95\% power and a 95\% confidence interval. Overall, a total of 1341 children $(n=743$ and 598 in 2009-2010 and 2014-2015, respectively) were evaluated in the post hoc analyses (Fig. 2).

\section{Enrolment of patients}

Standardized procedures and protocol were used at all sites. Briefly, patients were eligible for enrollment in the study if they were: aged 6-59 months, had symptoms compatible with acute uncomplicated malaria such as fever, anorexia, vomiting or abdominal discomfort with or without diarrhea with $P$. falciparum mono-infections from $\geq 1000 \mathrm{\mu l}^{-1}$ to $200000 \mathrm{\mu l}^{-1}$ of blood, a body (axillary) temperature $>37.4{ }^{\circ} \mathrm{C}$ or in the absence of measured fever, a recent history of fever in the 24 to $48 \mathrm{~h}$ before presentation, absence of other concomitant illness, no history of antimalarial drug ingestion in the 2 weeks prior to enrolment, no evidence of severe malaria $[17,18]$, and parents or guardians gave written informed consent. Using computer generated numbers, patients were allotted to AL or AA treatment groups prior to the commencement of recruitment into the study by personnel who was not involved in the selection and enrolment procedures. Sealed opaque envelopes containing the randomization were only opened by the attending

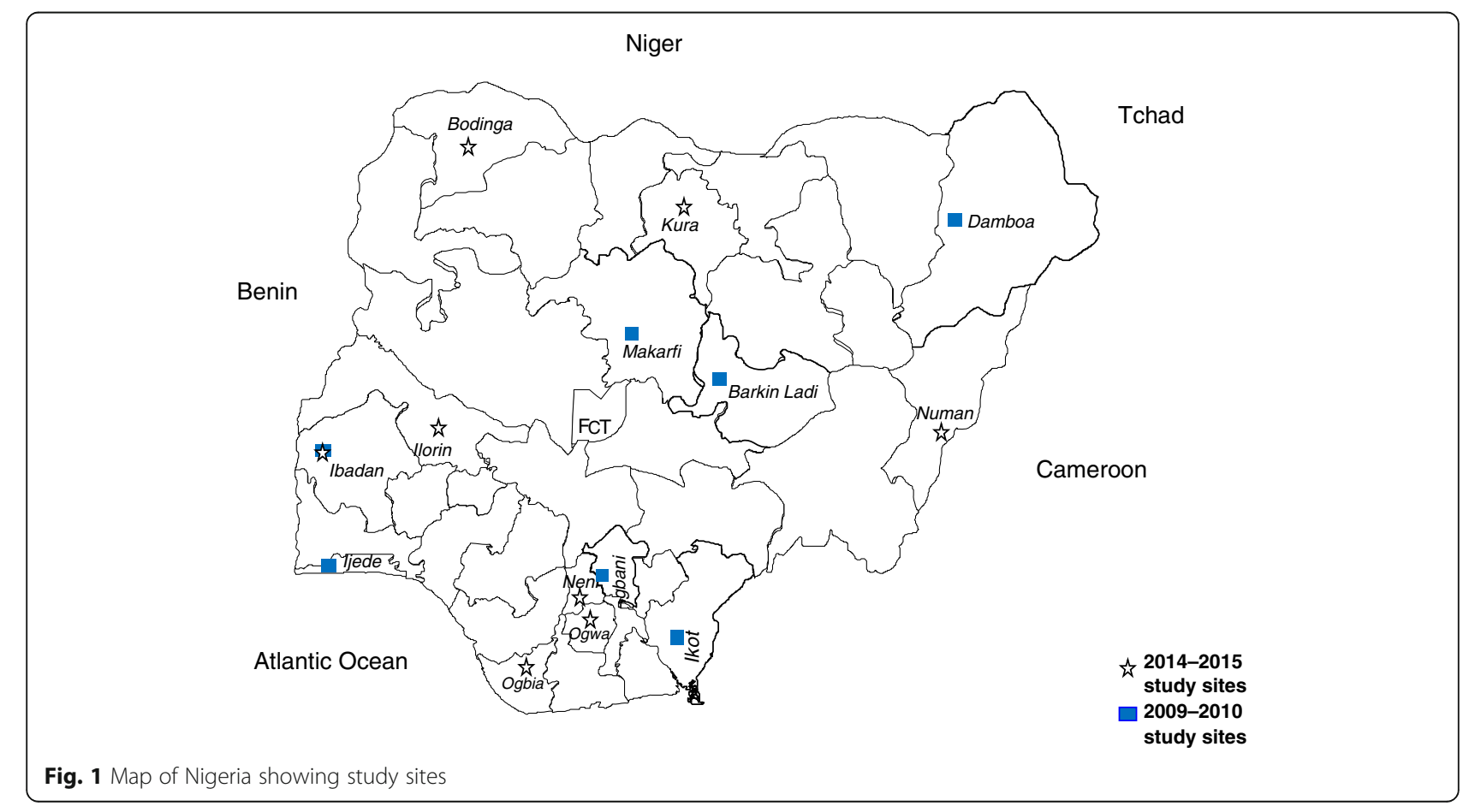




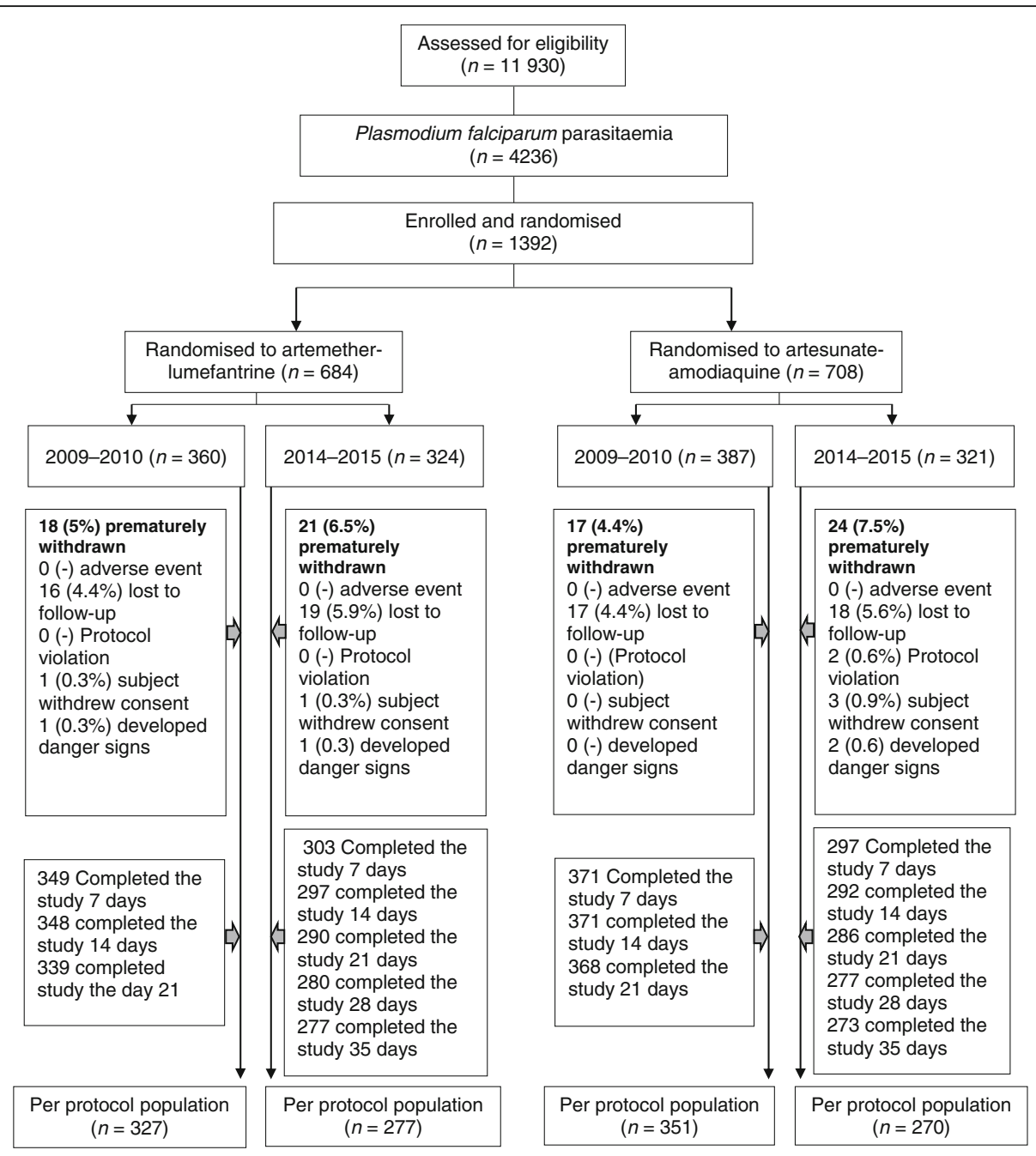

Fig. 2 Study profile of children randomised in efficacy study

physician at the time of recruitment. Treatment randomization was 1:1 for all patients at all sentinel sites during the two study periods.

\section{Study drug and administration}

Patients were administered with 3 days standard treatment of AL or AA as previously described $[15,16,19]$. Briefly, AL (Coartem ${ }^{\circ}$, Novatis, Basel, Switzerland) was given as follows: patients weighing $5-14 \mathrm{~kg}$ received one tablet, and those weighing $>14-24 \mathrm{~kg}$ received two tablets at presentation $(0 \mathrm{~h}), 8$ hours later and at 24, 36, 48 and $60 \mathrm{~h}$ after the first dose (each tablet of AL contains $20 \mathrm{mg}$ of artemether and $120 \mathrm{mg}$ of lumefantrine) while AA (Winthrop ${ }^{\circ}$, Sanofi Aventis, France) was given as follows: patients weighing $>4.5$ to $<9 \mathrm{~kg}$ received one tablet, those weighing $>9$ to $<18 \mathrm{~kg}$ received one tablet and those weighing $>18$ to $<24 \mathrm{~kg}$ received one tablet of the following formulations: $25 \mathrm{mg} / 67.5 \mathrm{mg}, 50 \mathrm{mg} / 135 \mathrm{mg}, 100 \mathrm{mg} /$ $270 \mathrm{mg}$ of fixed dose combination of artesunate/ amodiaquine, respectively daily for 3 days. All drugs were given orally. In children who were not able to swallow whole tablets, the tablets were carefully crushed using a tablet crusher, dissolved in water and administered orally. The three daily doses of AA, and that of AL at 0 (first dose at enrolment), eight, 24 and $48 \mathrm{~h}$ were given by direct observed therapy (DOT). The second daily doses of AL at 36 and $60 \mathrm{~h}$ after the first dose were given by guardians or parents of the children at home. A phone call was made to remind guardians/parents of time of the second daily doses of AL and to monitor the outcome of drug administration. A full replacement of dose was administered if vomiting occurred within $30 \mathrm{~min}$ of administration. Patients who vomited the dose after a repeated administration were excluded from the study.

\section{Study flow and clinical procedures}

The day of presentation (day of starting treatment) was regarded as day 0 . Thick and thin blood films, taken 
from a finger prick, were obtained from each child as soon as they came to the clinic and the slides were carefully labelled with the patients' codes and air-dried before being Giemsa-stained. Follow-up with clinical, parasitological and haematocrit evaluation was done daily on days 1-3 and 7, and thereafter, weekly for additional 3-5 weeks. If symptoms or parasitaemia was present on day 3, patients were also seen for assessment on day 4. Anaemia was defined as haematocrit $<30 \%$ and was classified as mild, moderate or severe if haematocrit value was $21-29,15-20$ or $<15 \%$, respectively.

\section{Parasitological assessment}

Parasitaemia, in Giesma-stained thick blood films, was estimated by counting asexual parasites relative to 500 leukocytes, or 500 asexual forms whichever occurred first. From this figure, the parasite density was calculated assuming a leukocyte count of $6000 \mathrm{\mu l}^{-1}$ of blood [20]. Presence of sexual forms was noted on blood slides but their densities were not quantified. A slide was considered asexual or sexual parasite negative if no parasite was detected after examination of 200 microscope fields. Asexual parasite reduction ratio (PRR) 1 or 2 days after treatment initiation (PRRD1 or PRRD2), and asexual parasite positivity 3 days after treatment initiation (APPD3), and parasite clearance time (PCT) determined by microscopy or estimation of haematocrit were defined or determined as previously described [16, 21]. Briefly, PRRD1 or PRRD2 was defined as the ratio of day 0/day 1 parasitaemia [that is, $P R R_{D 1}=\frac{\text { Parasitaemia on day } 0}{\text { Parasitaemia on day } 1}$ ] or ratio of day 0/day 2 parasitaemia [that is, $P R R_{D 2}=$ Parasitaemia on day 0 ], respectively; APPD1-3 as proportion of patients with residual parasitaemia on days 1 , 2 or 3 after treatment initiation, respectively; and PCT as the time elapsing from treatment initiation until complete clearance of peripheral parasitaemia.

\section{Parasite genotyping}

Finger pricked blood samples were spotted on 3MM Whatman $^{(\mathrm{R})}$ filter paper on days $0-3,7,14,21,28$, in 2009-2010, and additionally on days 35 and 42 in 20142015, and at the time of treatment failure for parasite genotyping. The filter papers were air-dried, labelled, and stored in individual envelope bags with desiccant in order to avoid moisture and fungi growth that could destroy the integrity of the collected samples until analyzed. PCR parasite genotyping pre- and post-treatment initiation was done using P. falciparum merozoite surface protein 1 or 2 (MSP 1 or MSP 2) or both genes as previously described $[15,16,22]$. Briefly, block 2 of the merozoite surface protein-1 (MSP-1) and the block 3 of the merozoite surface protein-2 (MSP-2) genes, were amplified by two rounds of polymerase chain reaction (PCR) using specific primers [23]. Five microliters of the nested PCR product were resolved by electrophoresis on a $2 \%$ agarose gel and sized against 100-basepair molecular weight DNA ladder (New England Biolabs, Beverly, MA).

Parasite DNA banding patterns 3 days after treatment initiation and that at the time of recurrent parasitaemia were compared side by side with the corresponding pretreatment DNA bands to detect actual delay in asexual parasite clearance and to compare parasite populations structures in samples, as well as to differentiate recrudescence from re-infection, respectively. Banding patterns were binned in $20 \mathrm{bp}$ using the GBox and the genetic analyzer software. Post-treatment and primary infection parasites showing identical banding patterns at both MSP-1 and MSP-2 loci were considered as recrudescence, whereas non-identity in banding patterns in at least one targeted locus of MSP-1 or MSP-2 was considered as newly acquired infections. In order to confirm the absence of recurrent parasitaemia, samples obtained from one in every four patients with microscopically negative blood films were also subjected to PCR analysis. An infection was considered polyclonal if it contained multiple clones (measured by amplicon fragment sizes) per allelic family on gel electrophoresis resolution of the parasite DNA. Multiplicity of infection was defined as the number of difference alleles per infection detected in positive samples of a population.

\section{Kinetic evaluation of time-course of parasitaemia following treatment initiation}

In the later studies (Clinical Trial Registration Numbers PACTR201508001188143, 3 July 2015; PACTR 201508001191898, 7 July 2015 and PACTR2015080 01193368, 8 July 2015) in 360 children aged 6-191 months enrolled in Ibadan site alone in parallel studies during the same time period (2009-2010 [ $n=254]$ and 2012-2015 [ $n=106])$, clinical and parasitological evaluations were done at the following times: pretreatment (0), 1, 2, 4, 6, 8 and $24 \mathrm{~h}$ and on days $2-7$, $14,21,28,35$ and 42 post-treatment initiation. The kinetics of the time-course of the asexual parasitaemia was estimated using a non-compartment model as previously described [16, 22, 24, 25]. Briefly, parasite densities (concentrations) versus time until complete clearance of parasitaemia were plotted on a semilogarithmic graph. Final parasite density at the time of apparent clearance was assumed to be 0.01 asexual parasites/ $\mu$ l of blood, a level below microscopic detection. The apparent terminal elimination rate constant $(\lambda)$ was obtained by least square regression analysis of the post peak log-linear part of the parasitaemia-time 
curve and apparent terminal elimination half-life of parasitaemia was obtained from $\ln 2 / \lambda$ (that is, $\lambda \mathrm{t}=$ 0.693).

\section{Data analysis}

Data were analyzed using version 6 of Epi-Info software (Centers for Disease Control and Prevention, Atlanta, GA, USA) [26] and the statistical program SPSS for Windows version 22.0 (SPSS Inc., Chicago IL, USA) [27]. Variables considered in the analysis were related to the densities of $P$. falciparum asexual forms. Proportions were compared by calculating $\chi^{2}$ using Yates' correction, Fisher's exact or Mantel Haenszel tests as appropriate. Normally distributed, continuous data were compared by Student's t test and analysis of variance (ANOVA). Post hoc comparisons of parameters between the two treatments, where necessary, were done using Tukey Honestly Significant Difference Test (Tukey HSD). Kaplan-Meier estimator and pair-wise log-rank tests were used to determine cumulative risk of recurrent parasitaemia on day 28, after the initial clearance of parasitaemia. Univariate analyses and stepwise multiple logistic regression models were used to test the association between demographic, clinical, parasitological or haematological parameters and parasite positivity rates one or 2 days post-treatment initiation and recurrent parasitaemia following treatments and the independent predictors of these parameters, respectively. Data were double entered serially using patients' codes and were only analysed at the end of the study. All test of significance were two-tailed and $P$ values $<0.05$ were taken to indicate significant differences.

\section{Results}

\section{Study cohort}

In the initial studies, between October 2009 and December 2015, we enrolled 1341 children for the efficacy studies (AL $[n=663]$ and AA $[n=678]$ ) (Table 1). One 34 months old male treated with AA had early treatment failure and another 31 months old female treated with AL developed danger signs within 1 day of enrolment in 2009-2010. In 2014-2015, 3 children had early treatment failure (two males aged 9 months and 24 months treated with AA and one female aged 24 months treated with AL) developed danger signs within 1 day of enrolment). Table 1 shows the baseline characteristics of the enrolled children according to treatment group and period of enrolment. Overall, children enrolled at the end of the second 5 years of deployment (2014-2015) were significantly older $(P<0.0001)$, had significantly lower body temperature $(P=0.005)$, significantly higher haematocrit $(P=0.04)$ and significantly lower proportion of children with gametocyte carriage $(P<0.0001)$ compared with those enrolled at the end of the first 5 years of deployment (2009-2010) (Table 1). Other parameters such as gender, weight, proportion with anaemia at presentation, enrolment parasitaemia were similar between the two enrolment periods.

\section{Transmission during period of observation}

Overall, parasite rate during the study period was $35.5 \%$ (4236 of 11930 children) and it did not differ during the two study periods (35\% [1826 of 5217 children] in 2009-2010 versus $35.9 \%$ [2410 of 6713 children] in 2014-2015). Gametocyte carriage declined significantly during the two study periods (see below). Data on other indices of transmission intensity during the period of observation were not evaluated.

\section{Clinical responses \\ Fever clearance}

Treatment with AA cleared fever significantly faster than AL [mean of 1.09 days ( $95 \%$ CI: $1.06-1.12, n=470$ ) versus mean of 1.2 days (95\% CI: $1.15-1.25, \quad n=462)$ respectively, $P<0.0001]$. Time to clear fever rose significantly $(P=0.002)$ from a mean of 1.1 days ( $95 \% C I$ : $1.08-1.14, n=555)$ in 2009-2010 to a mean of 1.2 days (95\% CI: $1.2-1.3, n=377$ ) in 2014-2015 (Table 2). Post hoc analysis showed an increase in fever clearance time in 2014-2015 in AL- $(P=0.002)$ but not in AA-treated children $(P=0.36)$ compared with 20092010 (Table 2).

\section{Adverse events}

The proportions of children reporting adverse events in the first week of initiating treatments were similar during the two time periods (78 of 223 children [35\%] versus 47 of 117 children [37\%] in 2009-2010 and 2014-2015, respectively, $P=0.75)$.

\section{Parasitological treatment responses Residual asexual parasitaemia 1 day after treatment initiation (APPD1)}

In all children, there was no expansion of baseline asexual parasitaemia 1 day after treatment initiation. When data from both treatment groups were pooled together, there was a striking increase in the proportion of children with residual asexual parasitaemia 1 day after treatment initiation between 2009 and 2010 and 2014-2015 (from 53.6\% [398 of 743 children] to 61.9\% [370 of 598 children], $P=0.002)$. The increase was significant with AA treatment (49\% [188 of 384 children] versus 58\% [171 of 295 children], $P=0.02$ ]) but not with AL treatment (58.3\% [210 of 360 children] versus $65.7 \%$ [199 of 303 children], $P=0.06)$. 


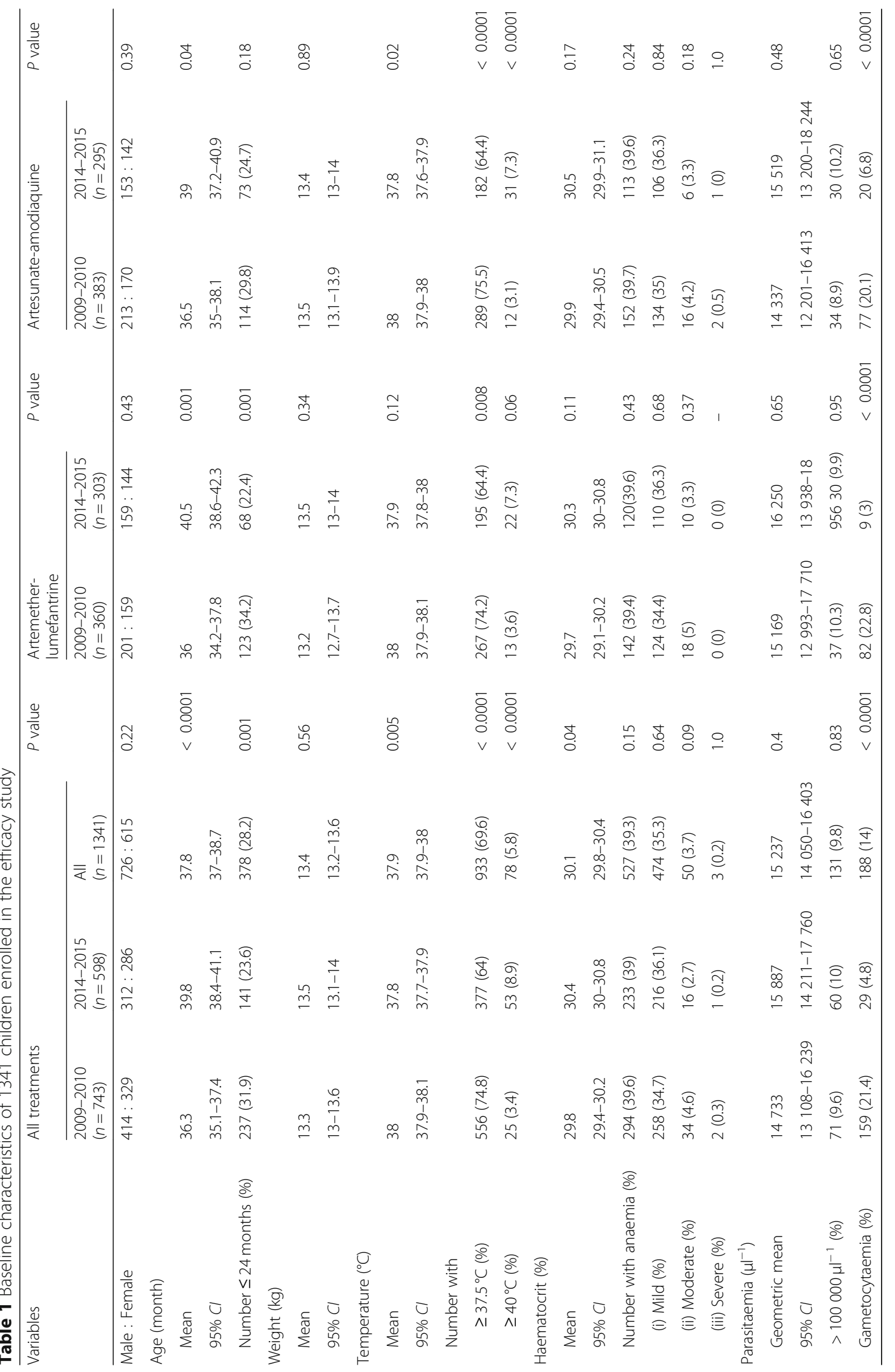




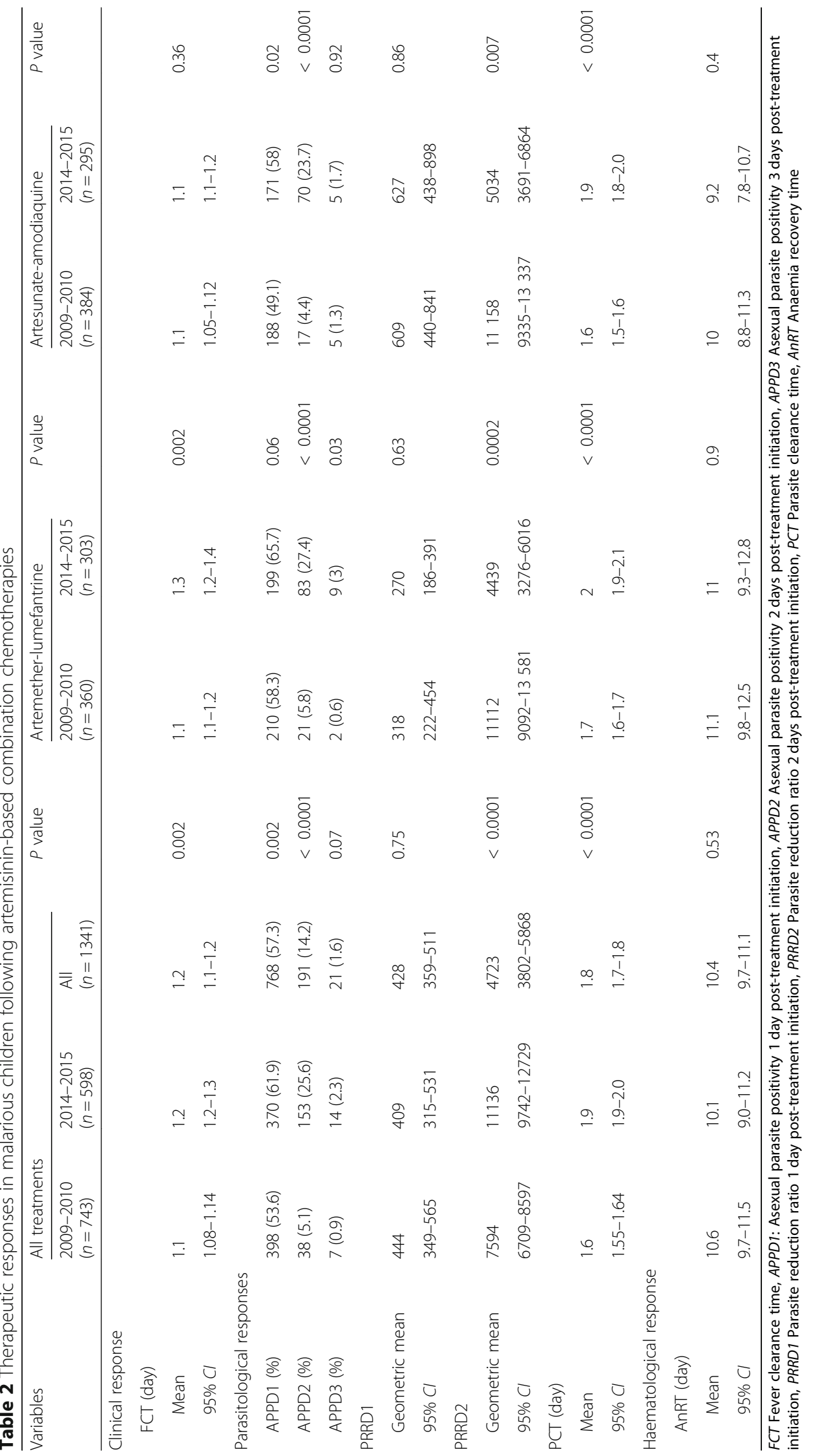


Table 3 Predictors of residual asexual parasitaemia 1 day post-initiation of artemisinin-based combination treatments in acutely malarious children

\begin{tabular}{|c|c|c|c|c|c|c|}
\hline Variable & Total number & Number with APPD1 & OR $(95 \% C l)$ & $P$ value & $\mathrm{aOR}(95 \% \mathrm{Cl})$ & $P$ value \\
\hline \multicolumn{7}{|l|}{ Gender } \\
\hline Female & 615 & 339 & 1 & & & \\
\hline Male & 726 & 429 & $1.2(0.9-1.5)$ & 0.16 & - & - \\
\hline \multicolumn{7}{|l|}{ Age (month) } \\
\hline$>24$ & 963 & 550 & 1 & & & \\
\hline$\leq 24$ & 378 & 218 & $1.0(0.8-1.3)$ & 0.9 & - & - \\
\hline \multicolumn{7}{|c|}{ Temperature at presentation $\left({ }^{\circ} \mathrm{C}\right)$} \\
\hline$\leq 37.4$ & 409 & 234 & 1 & & & \\
\hline$>37.4$ & 932 & 534 & $1.0(0.8-1.3)$ & 1.0 & - & - \\
\hline \multicolumn{7}{|l|}{ Fever on day 1} \\
\hline Absent & 1189 & 712 & 1 & & & \\
\hline Present & 132 & 82 & $1.2(0.8-1.8)$ & 0.33 & - & - \\
\hline \multicolumn{7}{|c|}{ Haematocrit at presentation (\%) } \\
\hline$<30$ & 728 & 425 & 1 & & & \\
\hline$\geq 30$ & 526 & 288 & $0.9(0.7-1.2)$ & 0.22 & - & - \\
\hline \multicolumn{7}{|c|}{ Haematocrit on day 1 (\%) } \\
\hline$\leq 27$ & 275 & 140 & 1 & & 1 & \\
\hline$>27$ & 640 & 384 & $1.4(1.1-1.9)$ & 0.01 & $1.6(1.2-2.1)$ & 0.002 \\
\hline \multicolumn{7}{|c|}{ Enrolment Parasitaemia $\left(\left.\mu\right|^{-1}\right)$} \\
\hline$\leq 75000$ & 1148 & 631 & 1 & & 1 & \\
\hline$>75000$ & 193 & 137 & $2.0(1.3-3)$ & $<0.0001$ & $1.9(1.3-2.7)$ & 0.002 \\
\hline \multicolumn{7}{|c|}{ Gametocytaemia } \\
\hline Absent & 1029 & 593 & 1 & & & \\
\hline Present & 188 & 115 & $1.2(0.8-1.6)$ & 0.41 & - & - \\
\hline \multicolumn{7}{|c|}{ Drug treatment } \\
\hline AA & 678 & 359 & 1 & & & \\
\hline $\mathrm{AL}$ & 663 & 409 & $1.4(1.2-1.8)$ & 0.001 & $1.8(1.1-2.8)$ & 0.01 \\
\hline \multicolumn{7}{|c|}{ Period of enrolment } \\
\hline 2009-2010 & 743 & 398 & 1 & & & \\
\hline 2014-2015 & 598 & 370 & $1.4(1.1-1.8)$ & 0.003 & $1.8(1.4-2.4)$ & $<0.0001$ \\
\hline
\end{tabular}

APPD1 Asexual parasite positivity 1 day post-treatment initiation, OR Odd ratio, aOR Adjusted odd ratio, CI Confidence interval, $A L$ Artemether-lumefantrine, $A A$ Artesunate-amodiaquine

\section{Multivariate analysis of residual asexual parasitaemia (APPD1)}

In a multivariate logistic regression model, haematocrit $>27 \%$ 1 day after treatment initiation, enrolment parasitaemia $>75$ $000 \mathrm{\mu l}^{-1}$, treatment with AL and enrolment in 2014-2015 independently predicted residual APPD1 (aOR $\geq 1.6$ and $P \leq$ 0.01 , Table 3 ). When patients treated with $\mathrm{AL}$ were analysed separately, enrolment body temperature $>40^{\circ} \mathrm{C} \quad(\mathrm{aOR}=2.8$, 95\% CI: 1.1-6.9, $P=0.03$ ), enrolment haematocrit $>32 \%$ $(\mathrm{aOR}=1.4,95 \% \mathrm{CI}: 1.0-2.0, P=0.048)$ and asexual parasitaemia $>75000 \mu^{-1}(\mathrm{aOR}=1.7,95 \% C I: 1.0-2.9, P=0.04)$ independently predicted APPD1. When patients treated with AA were analysed separately, haematocrit $>27 \% 1$ day after treatment initiation $(\mathrm{aOR}=2.4,95 \% \mathrm{CI}: 1.6-3.7, P<0.0001)$, enrolment parasitaemia $>75000 \mu^{-1}(\mathrm{aOR}=1.9,95 \% \mathrm{CI}$ : 1.1-3.2, $P=0.02)$ and enrolment in 2014-2015 (aOR=1.8, 95\% CI: $1.2-2.7, P=0.002)$ independently predicted APPD1.

\section{Residual asexual parasitaemia 2 days after treatment initiation (APPD2)}

The proportion of children with residual asexual parasitaemia 2 days after treatment initiation rose from 5.1\% (38 of 743 children) in 2009-2010 to $25.6 \%$ (153 of 598 children) in 2014-2015 $(P<0.0001)$ (Table 2$)$. In a post hoc analysis, the proportions of children with APPD2 rose significantly from 5.8 to $27.4 \%$ and from 4.4 to $23.7 \%$ in $\mathrm{AL}$ - and AA-treated 
Table 4 Predictors of residual asexual parasitaemia 2 days post-initiation of artemisinin-based combination treatments in acutely malarious children

\begin{tabular}{|c|c|c|c|c|c|c|}
\hline Variable & Total number & Number with APPD2 & $O R(95 \% C l)$ & $P$ value & $\mathrm{aOR}(95 \% \mathrm{Cl})$ & $P$ value \\
\hline \multicolumn{7}{|l|}{ Gender } \\
\hline Female & 615 & 92 & 1 & & & \\
\hline Male & 726 & 99 & $0.9(0.7-1.2)$ & 0.54 & - & - \\
\hline \multicolumn{7}{|l|}{ Age [month] } \\
\hline$>24$ & 963 & 138 & 1 & & & \\
\hline$\leq 24$ & 378 & 53 & $1.0(0.7-1.4)$ & 0.95 & - & - \\
\hline \multicolumn{7}{|c|}{ Temperature at presentation $\left({ }^{\circ} \mathrm{C}\right)$} \\
\hline$\leq 40$ & 1263 & 172 & 1 & & & \\
\hline$>40$ & 78 & 19 & $2.0(1.2-3.5)$ & 0.01 & $1.0(0.5-1.9)$ & 0.97 \\
\hline \multicolumn{7}{|l|}{ Fever on day 1} \\
\hline Absent & 1189 & 152 & 1 & & & \\
\hline Present & 132 & 32 & $2.2(1.4-3.4)$ & 0.001 & $1.8(1.1-3.2)$ & 0.03 \\
\hline \multicolumn{7}{|c|}{ Haematocrit at presentation (\%) } \\
\hline$<30$ & 728 & 115 & 1 & & & \\
\hline$\geq 30$ & 526 & 65 & $0.8(0.5-1.0)$ & 0.1 & - & - \\
\hline \multicolumn{7}{|c|}{ Haematocrit on day 1 (\%) } \\
\hline$\leq 29$ & 405 & 58 & 1 & & 1 & \\
\hline$>29$ & 510 & 102 & $1.5(1.1-2.1)$ & 0.01 & $1.7(1.1-2.5)$ & 0.01 \\
\hline \multicolumn{7}{|c|}{ Enrolment Parasitaemia $\left(\left.\mu\right|^{-1}\right)$} \\
\hline$\leq 100000$ & 1211 & 162 & 1 & & 1 & \\
\hline$>100000$ & 130 & 29 & $1.9(1.2-2.9)$ & 0.008 & $1.4(0.8-2.5)$ & 0.22 \\
\hline \multicolumn{7}{|c|}{ Parasitaemia on day 1} \\
\hline Absent & 573 & 19 & 1 & & 1 & \\
\hline Present & 768 & 172 & $8.4(5.2-13.7)$ & $<0.0001$ & $9.3(5.2-16.6)$ & $<0.0001$ \\
\hline \multicolumn{7}{|c|}{ Gametocytaemia } \\
\hline Present & 188 & 13 & 1 & & & \\
\hline Absent & 1029 & 178 & $2.8(1.6-5.1)$ & $<0.0001$ & $1.4(0.5-3.5)$ & 0.53 \\
\hline \multicolumn{7}{|c|}{ Drug treatment } \\
\hline AA & 678 & 87 & 1 & & & \\
\hline $\mathrm{AL}$ & 663 & 104 & $1.3(0.9-1.7)$ & 0.16 & - & - \\
\hline \multicolumn{7}{|c|}{ Period of enrolment } \\
\hline 2009-2010 & 743 & 38 & 1 & & & \\
\hline 2014-2015 & 598 & 153 & $6.4(4.4-9.3)$ & $<0.0001$ & $3.0(1.7-5.1)$ & $<0.0001$ \\
\hline
\end{tabular}

APPD2 Asexual parasite positivity 2 days post-treatment initiation, OR Odd ratio, aOR Adjusted odd ratio, $C l$ Confidence interval, $A L$ Artemether-lumefantrine, $A A$ Artesunate-amodiaquine

children, respectively, $(P<0.0001$ for each, Table 2$)$ during the same period.

\section{Multivariate analysis of residual APPD2}

In a multivariate logistic regression model, fever 1 day after treatment initiation, haematocrit $>29 \% 1$ day after treatment initiation, APPD1, and enrolment in 20142015 independently predicted residual APPD2 (aOR > 1.6 and $P \leq 0.03$, Table 4]. When patients treated with AL were analysed separately, asexual parasitaemia 1 day after treatment initiation $(\mathrm{aOR}=8.5,95 \% C I$ : 4.0-18.1, $P<0.0001)$ and enrolment in 2014-2015 (aOR $=3.7$ (95\% CI: 2.1-6.5), $P<0.0001$ ) independently predicted APPD2. When patients treated with AA were analysed separately, haematocrit $>29 \% 1$ day after treatment initiation $(\mathrm{aOR}=2.6,95 \% C I$ : $1.4-4.7, P=0.002)$, residual asexual parasitaemia 1 day after treatment initiation (aOR $=10.8,95 \% C I$ : 4.8-24.4, $P<0.0001$ ) and enrolment in 2014-2015 (aOR $=5.2,95 \% \quad C I: 2.5-10.8$, $P<0.0001)$ independently predicted APPD2. 


\section{Residual asexual parasitaemia 3 days after treatment initiation (APPD3)}

Proportion of children with PCR-confirmed residual asexual parasitaemia 3 days after treatment initiation rose from $0.9 \%$ (seven of 743 children) in $2009-2010$ to $2.3 \%$ (14 of 598 children) in 2014-2015 (Table 2). In a post hoc analysis, the proportions of children with APPD3 rose significantly from 0.6 to $3 \%$ (six folds) in AL-treated children, $(P=0.03$, Table 2) but not in AA-treated children during the same period (five of 383 children [1.3\%] versus 5 of 295 children [1.7\%] in 2009-2010 and 2014-2015, respectively, $P=0.92$ ).

\section{Parasite reduction ratio 1 day after treatment initiation (PRRD1)} Geometric mean parasite reduction ratio 1 day after treatment initiation did not change over the two study periods (444 [95\% CI: 349-565, $n=743$ ] versus 409 [95\% CI: $315-531, n=598]$, respectively, $P=0.75$, in 20092010 and 2014-2015, respectively).

\section{Parasite reduction ratio 2 days after treatment initiation}

Overall for both treatments, geometric mean parasite reduction ratio 2 days after treatment initiation (GMPRRD2) dropped by $57.2 \%$ (2.3-fold lower) between 2009 and 2010 (geometric mean 11 000, 95\% CI: 9700-13000) and 20142015 (geometric mean 4700, 95\% CI: 3800-5900, $P<0.0001)$. GMPRRD2 in the AL treatment group dropped by $60 \%$ (2.5-fold lower) (geometric mean $11000,95 \%$ CI: 9100-14 000) and (geometric mean 4400, 95\% CI: 3300$6000, P=0.0002$ ) and in the AA treatment group by $54.5 \%$ (2.2-fold lower) (geometric mean 11 000, 95\% CI: 9300-13 000) and (geometric mean 5000, 95\% CI: 3700-6900, $P=$ 0.007) between 2009 and 2010 and 2014-2015 (Table 2).

\section{Parasite clearance}

Treatment with AA cleared asexual parasitaemia significantly faster than AL (mean of 1.7 days [95\% CI: 1.6-1.8, $n=678$ ] versus mean of 1.8 days [95\% CI: $1.8-1.9, n=663$ ], respectively, $P=0.006)$. Parasite clearance time increased significantly from a mean of 1.6 days (95\% CI: $1.55-1.64$, $n=743)$ in $2009-2010$ to a mean of 1.9 days (95\% CI: 1.9 $2.0, n=598)$ in 2014-2015 $(P<0.0001$, Table 2). Post hoc analysis showed parasite clearance time rose significantly in children treated with $\mathrm{AA}$ and $\mathrm{AL}$ during the same time period $(P<0.0001$ for each, Table 2$)$.

\section{Probability of reappearance of asexual parasitaemia following initial clearance}

By day 28 , in a pooled analysis of both treatments, probability of recurrent asexual parasitaemia following initial clearance was significantly higher in children enrolled in 20142015 compared with 2009-2010 (Log rank statistic $=14.12$, $P=0.0002$, Fig. 3). When patients treated with AL and AA

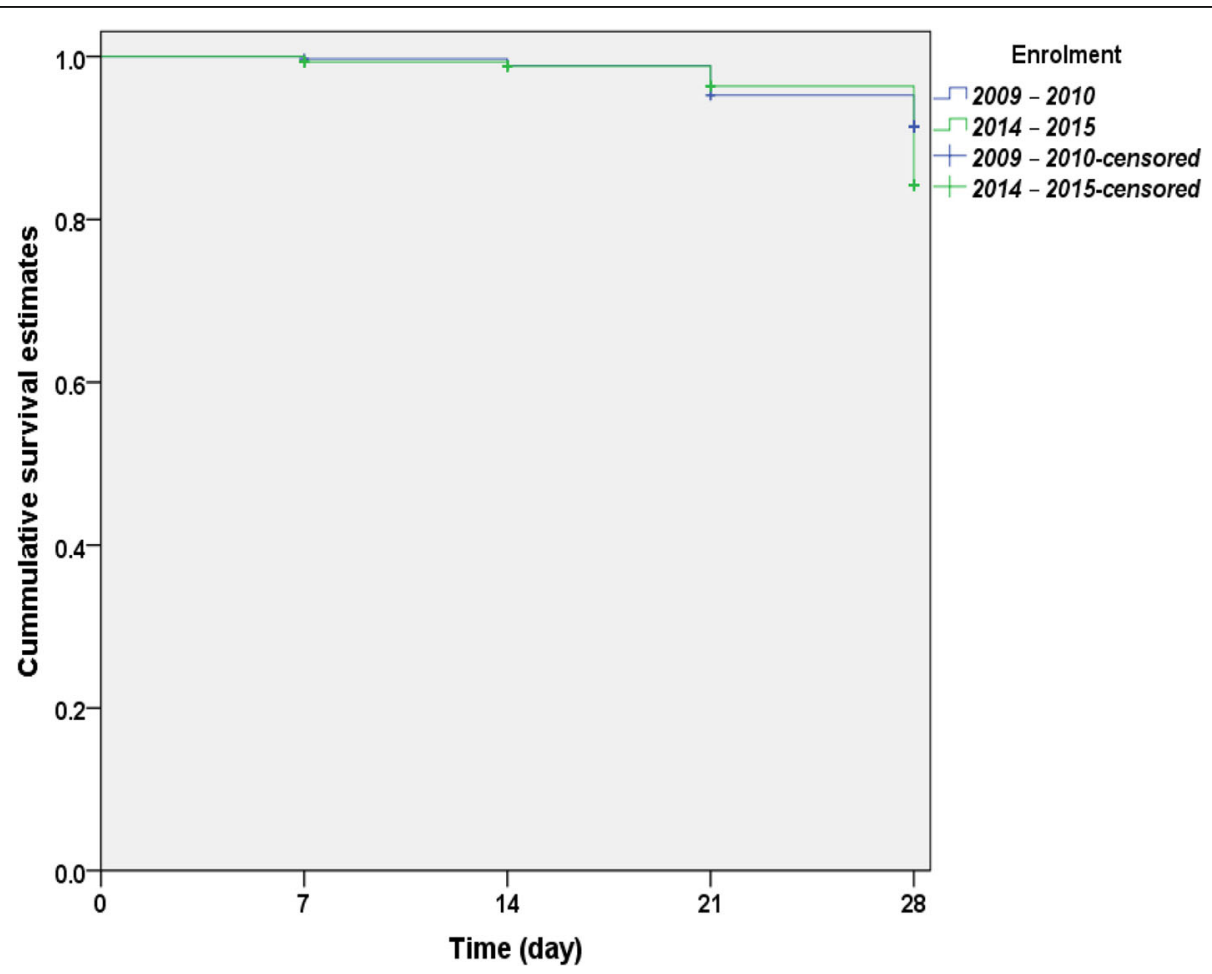

Fig. 3 Kaplan-Meier survival estimates of reappearance of asexual parasitaemia after initial clearance following artemisinin-based combination treatments in 2009-2010 (blue line) and 2014-2015 (green line). Log-rank statistic $=14.12, P=0.0002$. Pooled analysis of artemether-lumefantrine and artesunate-amodiaquine treatments 
were analysed separately, the probabilities of recurrent asexual parasitaemia were significantly higher in 2014-2015 compared with 2009-2010 (Log-rank statistic $=7.8$ and $5.64, P=0.020$ and 0.005 , respectively).

Multivariate analysis of recurrent asexual parasitaemia within 28 days after treatment initiation: In a stepwise multivariate logistic regression model of pooled data from the two treatment groups, residual asexual parasitaemia 1 day after treatment initiation (APPD1) $(\mathrm{aOR}=$ 1.7, 95\% CI: $1.2-2.5, P=0.005)$, gametocyte carriage within the first week of presentation $(\mathrm{aOR}=2.095 \% \mathrm{CI}$ : 1.3-3.8, $P=0.001)$ and enrolment in 2014-2015 (aOR= 2.0, 95\% CI: $1.4-2.9, P<0.0001)$ independently predicted recurrent asexual parasitaemia within 28 days of treatment initiation.

\section{Probability of recrudescent parasitaemia following initial clearance}

Recrudescent infections, confirmed by PCR, occurred in 34 of 731 children (4.7\%) in 2009-2010 and in 16 of 544 children $(2.9 \%)(P=0.16)$ by day 28 . In a pooled analysis of both treatments, probability of recrudescent parasitaemia following initial clearance was similar in children enrolled in 2009-2010 and in 2014-2015 (Log rank statistic $=2.78, P=0.1)$. When patients treated with $\mathrm{AL}$ and AA were analysed separately, the probabilities of recrudescent parasitaemia were similar at the two time periods (Log-rank statistic $=1.14$ and 1.71, $P=0.29$ and 0.19 , respectively).

\section{Multiplicity of infection pre-treatment initiation}

Overall, data for multiplicity of infection were available in 113 children (50 children in 2009-2010 and 63 in 20142015) who had recurrent parasitaemia. Proportion of children with polyclonal infection increased significantly from $32 \%$ in $2009-2010$ (16 of 50 children) to $57 \%$ in $2014-2015$ (36 of 63 children) $(P=0.01)$. Multiplicity of infection (MOI) also increased significantly from 1.3 (95\% CI: 1.2-1.5) to 2.0 (95\% CI: 1.7-2.3) $(P<0.0001)$ during the same time period.

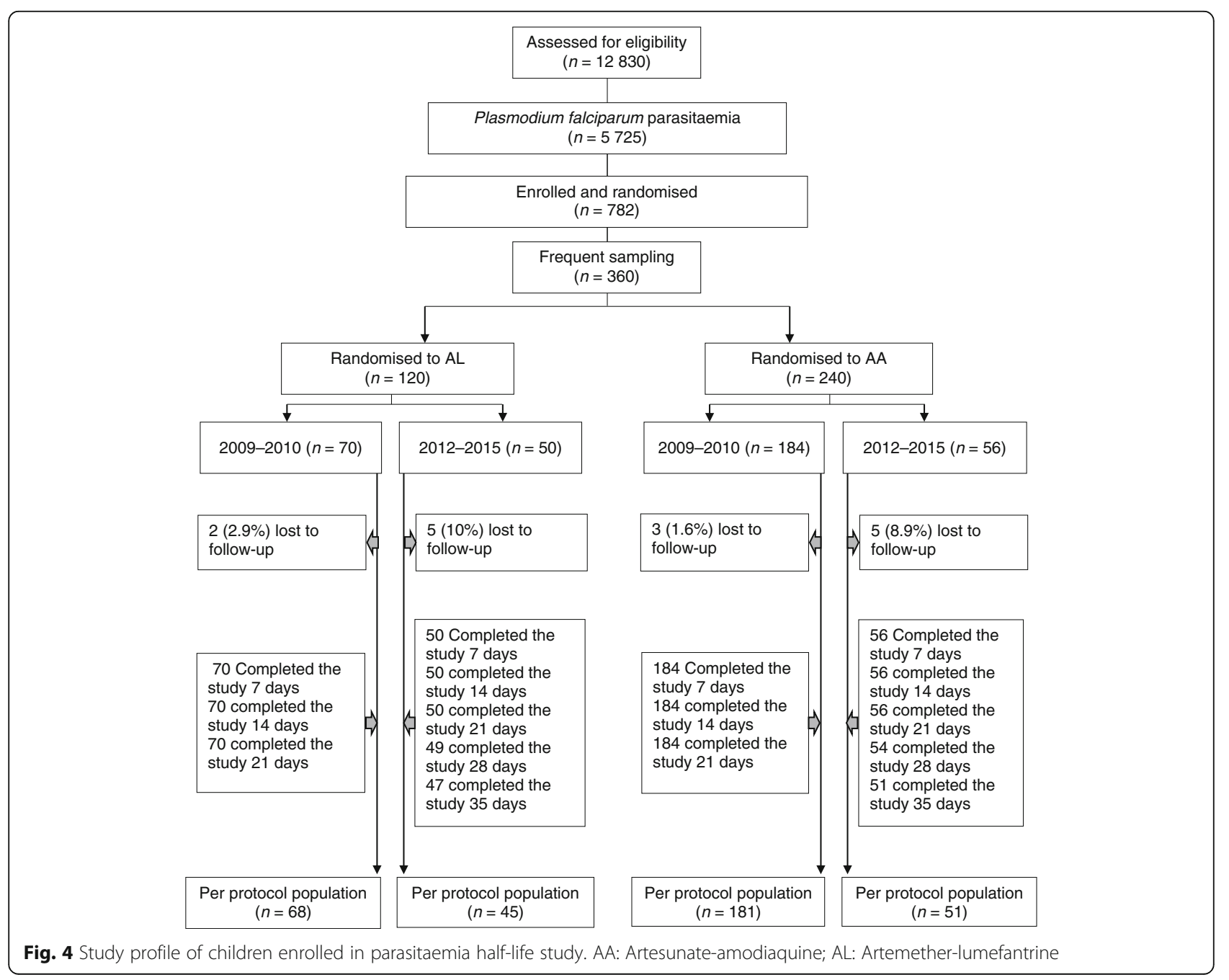


Kinetic evaluation of time-course of asexual parasitaemia following treatment initiation

\section{Study cohort}

Between May 2009 and July 2015 at Ibadan study site, we enrolled and randomized additional 360 children for kinetic evaluation of the time-course of parasitaemia at a ratio of 1:2 for AL and AA (AL $[n=120]$ and AA $[n=$ 240]) (Fig. 4). All children had follow-up record till day
7 and were included in the analysis. At presentation, children enrolled in 2012-2015 had significantly lower body temperature $(P=0.009)$, and geometric mean asexual parasitaemia $(P<0.0001)$ compared with those enrolled in 2009-2010 (Table 5). Parasite clearance time and residual asexual parasitaemia 1 day after treatment initiation were significantly higher and PRRD1 and PRRD2 significantly lower in children enrolled in 2012-

Table 5 Baseline characteristics and treatment responses of 360 children enrolled in the parasitaemia elimination half-life study

\begin{tabular}{|c|c|c|c|c|}
\hline Variables & $\begin{array}{l}2009-2010 \\
(n=254)\end{array}$ & $\begin{array}{l}2012-2015 \\
(n=106)\end{array}$ & $\begin{array}{l}\text { All } \\
(n=360)\end{array}$ & $P$ value \\
\hline \multicolumn{5}{|l|}{ Baseline characteristics } \\
\hline Male : Female & $141: 113$ & $53: 53$ & $194: 166$ & 0.4 \\
\hline \multicolumn{5}{|l|}{ Age (month) } \\
\hline Mean & 7.3 & 7.5 & 7.4 & 0.64 \\
\hline $95 \% \mathrm{Cl}$ & $6.9-7.7$ & $6.8-8.1$ & $7.0-7.7$ & \\
\hline Number 5 years (\%) & $54(21.3)$ & $22(20.8)$ & $76(21.1)$ & 1.0 \\
\hline \multicolumn{5}{|l|}{ Temperature $\left({ }^{\circ} \mathrm{C}\right)$} \\
\hline Mean & 38.4 & 38 & 38.3 & 0.009 \\
\hline $95 \% \mathrm{Cl}$ & $38.2-38.5$ & $37.8-38.3$ & $38.2-38.4$ & \\
\hline \multicolumn{5}{|l|}{ No. with } \\
\hline$\geq 37.5^{\circ} \mathrm{C}(\%)^{*}$ & $196(77.2)$ & $67(23.6)$ & $263(73.1)$ & 0.01 \\
\hline$\geq 40^{\circ} \mathrm{C}(\%)$ & $26(10.2)$ & $4(3.8)$ & $30(8.3)$ & 0.06 \\
\hline \multicolumn{5}{|l|}{ Haematocrit (\%) } \\
\hline Mean & 32.1 & 32.1 & 32.1 & 0.98 \\
\hline $95 \% \mathrm{Cl}$ & $31.6-32.6$ & $32.1-33.0$ & $31.7-32.6$ & \\
\hline No with anaemia (\%) & $60(23.6)$ & $25(23.6)$ & $85(23.6)$ & 1.0 \\
\hline \multicolumn{5}{|l|}{ Parasitaemia $\left(\left.\mu\right|^{-1}\right)$} \\
\hline Geometric mean & 71393 & 41404 & 60811 & $<0.0001$ \\
\hline Range & 1800-1096 636 & $2220-454875$ & 1800-1096 636 & \\
\hline$>100000 \mu \mathrm{I}^{-1}(\%)$ & $165(65)$ & $83(78.3)$ & $248(68.9)$ & 0.02 \\
\hline Gametocytaemia (\%) & $7(2.8)$ & $2(1.9)$ & $9(2.5)$ & 1.0 \\
\hline \multicolumn{5}{|l|}{ Treatment responses } \\
\hline \multicolumn{5}{|l|}{ Fever clearance time (day) } \\
\hline Mean $(95 \%$ Cl) & $1.1(1.0-1.1)$ & $1.1(1.0-1.2)$ & $1.1(1.05-1.13)$ & 0.26 \\
\hline \multicolumn{5}{|c|}{ Parasite clearance time (day) } \\
\hline Mean $(95 \%$ Cl) & $1.1(1.1-1.2)$ & $1.4(1.3-1.5)$ & $1.2(1.2-1.3)$ & $<0.0001$ \\
\hline APPD1 (\%) & $28(11)$ & $37(34.9)$ & $65(18.1)$ & $<0.0001$ \\
\hline APPD2 (\%) & $4(1.6)$ & $2(1.9)$ & $6(1.7)$ & 1.0 \\
\hline APPD3 (\%) & $3(1.2)$ & $0(0)$ & $3(0.8)$ & - \\
\hline \multicolumn{5}{|c|}{ Parasite reduction ratio 1 day } \\
\hline Geometric mean & $3.3 \times 10^{4}$ & $3.9 \times 10^{3}$ & $1.7 \times 10^{4}$ & $<0.0001$ \\
\hline Range & $2.7 \times 10^{0}-1.1 \times 10^{6}$ & $8.0 \times 10^{-1}-2.4 \times 10^{5}$ & $8.0 \times 10^{-1}-1.1 \times 10^{6}$ & \\
\hline \multicolumn{5}{|c|}{ Parasite reduction ratio 2 days } \\
\hline Geometric mean & $6.6 \times 10^{4}$ & $3.8 \times 10^{4}$ & $5.6 \times 10^{4}$ & $<0.0001$ \\
\hline Range & $1.6 \times 10^{1}-1.1 \times 10^{6}$ & $1.8 \times 10^{3}-3.6 \times 10^{5}$ & $1.6 \times 10^{1}-1.1 \times 10^{6}$ & \\
\hline
\end{tabular}


2015 compared with those enrolled in 2009-2010 $(P<0.0001$ for each) (Table 5). Proportion of children with asexual parasite clearance time of 1 day declined significantly from $89 \%$ (226 of 254 children) in 20092010 to $65.1 \%$ (69 of 106 children) in 2012-2015 $(P<0.0001)$ and proportions of children with asexual parasite clearance time of 2 days increased significantly from 9.5\% (24 of 254 children) to 33\% (35 of 106 children $)(P<0.0001)$ during the same time period (Fig. 5a). The frequency distribution of parasite clearance time during the two periods was unimodal.

\section{Terminal elimination half-time of asexual parasitaemia} Estimated terminal elimination half-time of asexual parasitaemia increased significantly from a mean of $1.1 \mathrm{~h}$ (95\% CI: $1.1-1.2, n=254)$ in $2009-2010$ to $1.3 \mathrm{~h}(95 \%$

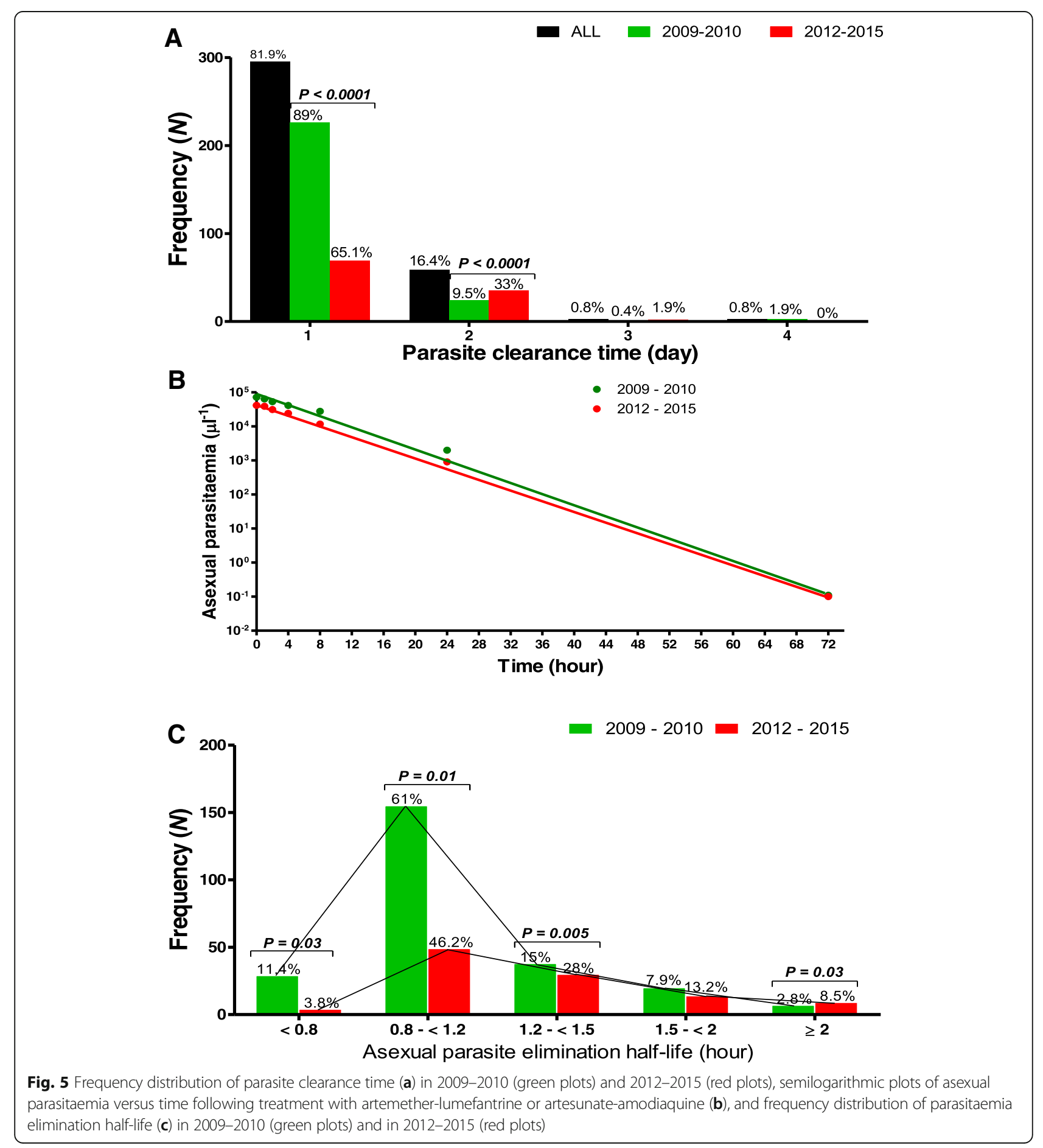


CI: $1.2-1.4, n=106)$ in $2012-2015(P<0.0001)$ (Fig. $5 \mathrm{~b})$. When analysed according to age groups, in 6-59 month-olds, terminal elimination half-time of asexual parasitaemia increased significantly from a mean of $1.1 \mathrm{~h}$ (95\% CI: $1.1-1.2, n=73)$ in $2009-2010$ to $1.4 \mathrm{~h}(95 \% \mathrm{CI}$ : $1.3-1.6, n=27)$ in 2012-2015 $(P=0.004)$. In children older than 59 months, terminal elimination half time of asexual parasitaemia also increased significantly from a mean of $1.1 \mathrm{~h}$ (95\% CI: $1.1-1.2, n=181)$ in $2009-2010$ to $1.3 \mathrm{~h}(95 \% \mathrm{CI}: 1.2-1.4, n=79)$ in $2012-2015(P=$ $0.001)$. There was no difference in mean terminal elimination half-times of asexual parasitaemia in the $<5$ and $>5$ year-olds $(P=0.68)$. Mean estimated terminal elimination half-times of asexual parasitaemia was similar in the two treatment groups $(1.2 \mathrm{~h}$ [95\% CI: 1.1-1.2, $n=120]$ versus $1.2 \mathrm{~h}$ [95\% CI: $1.1-1.2, n=240]$ in AL and AA, respectively, $P=0.94)$. There was a significantly positive correlation between parasite clearance time and terminal elimination half-time of asexual parasitaemia $(\mathrm{r}=0.64, P<0.0001, n=360)$.

\section{Frequency distribution of terminal elimination half-times of asexual parasitaemia and parasite clearance times}

The frequency distribution of terminal elimination half times of asexual parasitaemia was unimodal during the two study periods (Fig. 5c) as was the frequency distribution of parasite clearance times (Fig. 5a). In a pooled analyses, proportion of children with terminal elimination half-times of asexual parasitaemia $\geq 2 \mathrm{~h}$ increased significantly from $2.8 \%$ (7 of 254 children) in $2009-2010$ to $8.5 \%$ (9 of 106 children) in $2012-2015$ ( $P=0.03$, Fig. $5 \mathrm{c})$ and proportions of children with terminal elimination half-times of asexual parasitaemia $<1.2 \mathrm{~h}$ decreased significantly $(P<0.0001)$ from $72.4 \%$ (184 of 254 children) to $50 \%$ (53 of 106 children) during the same time period (Fig. 5c). One 60 months old male had a terminal elimination half-time of asexual parasitaemia of $3 \mathrm{~h}$.

In a univariate analyses to determine the factors associated with an terminal elimination half-time of asexual parasitaemia $>1.5 \mathrm{~h}$, namely gender (male or female), age $<5$ or $>5$ years, body temperature $<37.4{ }^{\circ} \mathrm{C}$ or $>$ $37.4{ }^{\circ} \mathrm{C}$, presence or absence of fever 1 day after treatment initiation, haematocrit $<30 \%$ or $\geq 30 \%$, enrolment parasitaemia $\leq 100000 \mu^{-1}$ or $>100000 \mu^{-1}$, treatment with AL or AA, enrolment in 2009-2010 or 2012-2015, only enrolment in 2012-2015 was significantly associated with a terminal elimination half-time of asexual parasitaemia $\geq 1.5 \mathrm{~h}$ (27 of 254 [11\%] versus 23 of 106 [22\%], OR $=2.3,95 \% C I: 1.3-4.3, P=0.009]$.

\section{Discussion}

In these country-wide studies of the efficacies of artemisinin-based combination therapies during a 10-year period of deployment as first-line antimalarials in Nigeria, we report a significant decline of early responses of childhood P. falciparum infections to AL and AA. Parasite positivity 1 day after treatment initiation increased insignificantly from 58 to $66 \%$ over time in children receiving $\mathrm{AL}$ and significantly from 50 to $58 \%$ over time in those receiving AA. In addition, parasite positivity 2 days after initiating both treatments increased significantly from 5 to $26 \%$ over time. These increases indicate declining or worsening of early response markers, which are thought to be primarily dependent on the artemisinin components of ACTs [28, 29]. Although the magnitude of the decline in early response markers is much smaller than in the GMS $[1-7,30]$, the substantially increased APPD3 over time in those treated with AL is reminiscent of the situation in the GMS where declining responsiveness was a prelude to development of artemisinin-resistance in P. falciparum [3, 30]. For both ACTs, the substantial increase in APPD3 from 0.9 to $2.3 \%$ ( 2.6 folds) over time is striking and calls for a formal evaluation, in vivo, of artemisinin efficacy test of delayed parasite clearance that is devoid of confounders by artemisinin derivatives partner drugs in children under 5-year-old from these endemic areas as was carried out in some malaria transmission settings $[9,30]$.

Increment in APPD1 over time is common to all areas with declining responsiveness in P. falciparum to ACTs $[2,30]$. However, it is unclear why we did not observe a significant increase in APPD1 in children treated with AL. One possible reason for this observation is that the relatively long period between deployment in 2005 and our first study in 2009-2010 masked the incremental change which probably occurred earlier than 2009-2010. To support this explanation, are the findings both APPD1 and terminal elimination half-time of asexual parasitaemia increased significantly with both ACTs when interval between the study periods was only 2 years (Table 5 and Fig. 5b).

There was remarkable similarity between some of the independent predictors of APPD1 and APPD2 such as haematocrit $\geq 27 \%$ and enrolment in 2014-2015 but there was no expansion of baseline asexual parasitaemia, stable baseline asexual parasitaemia, or less than $75 \%$ decrease in baseline asexual parasitaemia that would have indicated marked reduction of response to the two ACTs in any of the patients 1 day after treatment initiation. It is surprising that in contradistinction to the situation in some countries in the GMS, where declining responsiveness is associated with increased gametocyte carriage and transmission of parasites with reduced susceptibilities to ACTs [3], gametocyte carriage decreased significantly during the period of deployment in the cohorts of children we evaluated. This is explicable in the context of small but significant decline in responsiveness 
overtime that has not reached the critical stage for the emergence of resistance and its associated increasing gametocyte carriage. However, gametocytaemia within 1 week of initiating treatment and enrolment in 20142015 independently predicted recurrent asexual parasitaemia within 28 days of treatment initiation. These findings would suggest declining gametocyte carriage over time alone would be insufficient to prevent transmission of gametocytes arising from parasites with reduced susceptibility should resistance develop in the future to any of the two ACTs evaluated. It would also appear from the results of the multiple logistic regression models, enrolment in 2014-2015 may be synonymous with declining responsiveness to the two ACTs. This is not surprising as 2014-2015 may be a watershed to progression or otherwise of reduced susceptibility.

Parasites with alleles that confer slow clearance of parasitaemia in the GMS notably PfK13 C580Y, R539T, $\mathrm{Y} 493 \mathrm{H}$ are not frequently encountered in Africa [10,31]. However, the observation of significantly increased parasite clearance time over time raises concerns about possible emergence with time of parasites with slow clearance phenotypes similar or dissimilar to those in the GMS. Thus, it is possible, with time, parasites with slow clearance phenotypes with alleles different from those in the GMS may emerge from indigenous $P$. falciparum populations in Africa as has been recently described in a non-immune visitor to Equatorial Guinea [32].

Four points may help explain the possibility of emergence with time of parasites with slow clearance phenotypes in endemic areas of Nigeria: First, an increase in APPD3 from a baseline of 0.9 to $2.3 \%$ over time is unlikely to be a random phenomenon. Second, the significantly increased risk of recurrent parasitaemia overtime, in the presence of the long acting partner drugs, may contribute to parasite survival in the presence of less than optimal parasiticidal drug concentrations [33, $34]$, even when PCR-corrected 28 days efficacy rates are high. Third, in many endemic areas as opposed to areas of low transmission, parasite burdens in less than 5-year olds and the probabilities of low dosing which may permit de novo selection of resistant parasites are high [33, $34]$. Finally, a $20 \%$ increase in terminal elimination halftime of asexual parasitaemia in 2 years in these endemic areas of full ACTs sensitivity [15, 16, 21], when combined with significantly increased risk of recurrent parasitaemia may signal downward spiral of the declining responsiveness within a relatively short time frame.

Estimation of parasite clearance half-life is thought to be the best in vivo measure of artemisinin drug effect [29]. Although the significant increments in terminal elimination half-times of asexual parasitaemia over time in the cohorts of both young and older children we evaluated were not as marked as those reported from GMS $[5,6,30]$, the data provided compelling evidence of declining parasite clearance rate manifested as significant increases in both the frequency of terminal elimination half-times of asexual parasitaemia $\geq 2 \mathrm{~h}$ from 2.8 to $8.5 \%$, and significant reduction of frequency of terminal elimination half times of asexual parasitaemia of $\leq 0.8 \mathrm{~h}$ from 11 to $4 \%$ within a short period of 2 years (Fig. 5c). In many GMS countries, where resistance to artemisinin has developed, for example in southern Myanmar [5, 30], frequency distribution of parasite clearance time and terminal elimination half-life of parasitaemia is bimodal. The unimodal frequency distribution of terminal elimination half-times of asexual parasitaemia in the cohort of children we evaluated supports an earlier report of apparent absence of parasites with slow clearance phenotype in endemic areas of Nigeria [10]. However, it is plausible to expect that with increasing 'right shift' in frequency distribution of terminal elimination half-times of asexual parasitaemia over time, it is likely a bimodal distribution will emerge in the future with slow clearance parasite phenotypes.

In order not to overestimate terminal elimination halftimes of asexual parasitaemia or misinterpret the observed unimodal frequency distribution of terminal elimination half-times of asexual parasitaemia, frequent samples were obtained from the patients. The limitations of our study in this context are the non-estimation of concentrations of the artemisinin components of the administered ACTs and/or their metabolites, and apart from age, the non-evaluation of the many host factors that may influence parasite clearance times such as immune status. ACTs may mobilize asexual parasites from the deep tissue into peripheral blood in the early hours following initiation of treatments in drug sensitive infections in children from endemic areas of Africa [9, 22]. This action may increase parasite clearance time when compared with those without mobilization [22]. It is unclear how this phenomenon would influence terminal elimination half-times of asexual parasitaemia in those with this phenomenon; we did not evaluate this phenomenon in our post hoc analyses.

Individual analysis of the two studies (2009-2010 and 2014-2015) showed spatial heterogeneity in clearance indices. For example, for both studies, recrudescence and residual parasitaemias on days 1 and 2 were significantly higher on the eastern compared with western flank of the study sites $[15,16]$. In addition, if resistance were to develop in the future, it would more like emerge from the eastern compared to the western flank of the study sites and to occur in AL- compared to AA-treated children. However, our post hoc analyses of the two studies were not sufficiently powered to detect spatial heterogeneity of the clearance indices. 
Although we did not evaluate immunity in the cohorts of children in the post hoc analyses, the limited data on transmission intensity suggested this did not change over time. The significantly increased rate of polyclonal infections and multiplicity of infection in the absence of presumed decrease in immunity, may support, in part, the emergence of a population of parasites with reduced susceptibility to the two ACTs. In a similar study of two ACTs in similarly aged Kenyan children, in an area of seasonal but intense transmission, Borrmann and others [12] found that significant decline in responsiveness over a relatively short period of time was accompanied by decrease in immunity of the children.

Overall, the critical questions are: are the present observations of post hoc analyses, an unmasking of innate reduced in vitro susceptibility of $P$. falciparum isolates in Nigeria to artemisinin first reported in the 1992, 13 years before the adoption and deployment of artemisinin-based combination therapies [35]? Is a relatively recent report of parasite isolates with reduced in vitro susceptibility to artemether from the same endemic area and their association with transporter genes [36] an indirect confirmation of the 1992 observation? Taken together, the earlier observations support the present observations of post hoc analyses, and all indicate the emergence of clonal predominance of parasites with reduced susceptibility to artemisinin components of the two ACTs evaluated.

\section{Conclusions}

In conclusion, the declining parasitological responses through time to artemether-lumefantrine and artesunateamodiaquine raises concerns and may be due to predominance of parasites with reduced susceptibility or decreasing herd immunity in young children in endemic areas of Nigeria.

\section{Additional file}

Additional file 1: Multilingual abstracts in the five official working languages of the United Nations. (PDF $259 \mathrm{~kb}$ )

\section{Abbreviations \\ AA: Artesunate-amodiaquine; ACT: Artemisinin-based combination therapy; AL: Artemether-lumefantrine; AnRT: Anaemia recovery time; AOR: Adjusted odd ratio; APPD1: Asexual parasite positivity one day post-treatment initiation; APPD2: Asexual parasite positivity two days post-treatment initiation; APPD3: Asexual parasite positivity three days post-treatment initiation; Cl: Confidence interval; DNA: Deoxyribonucleic acid; GMS: Greater Mekong Subregion; MOI: Multiplicity of Infection; MSP-1: Merozoite surface protein-1; MSP-2: Merozoite surface protein-2; OR: Odd ratio; PCR: Polymerase chain reaction; PCT: Parasite clearance time; PRR: Parasite reduction ratio; PRRD1: Parasite reduction ratio one day post-treatment initiation; PRRD2: Parasite reduction ratio two days post-treatment initiation}

\section{Acknowledgements}

We thank the parents/guardians of the children and the children for their participation in the study. We also thank the Medical Officers at the recruiting facilities for assisting with patient recruitment, and the State Government where the sentinel sites are located for providing logistic support.

\section{Authors' contributions}

AS led the design, conduct, data analyses and manuscript preparation and was the National Principal Investigator. GN, KA, FOI, AIA performed kinetics study. GN, KA, CA, GOG participated in data analysis and manuscript preparation. KA, OAF, CTH performed parasite genotyping and manuscript preparation; SO, HUO, MM, PA, WO, IW, OM, FF, JCE, NJ, JA, RW, GE were Sentinel Sites Principal Investigators and were involved with data collection and analysis. WO, FU were involved with study design. TA, TMD, OTA, SA, OKB, OAW, CO, OA, BF, EOA were involved with kinetics study. NME and AO were involved with study design. All authors read and approved the final draft of the manuscript.

\section{Funding}

The efficacy study from which the data were derived received financial support from Global Fund for AIDS, Tuberculosis and Malaria, United States President's Malaria Initiative (USPMI), and Malaria Consortium Grants to The Federal Ministry of Health, Abuja, through Drug Therapeutic Efficacy Testing in Nigeria. The molecular aspects of the study were supported by funding from the National Human Genome Research Institute, National Institutes of Health, Department of Health and Human Services, under Grant Numbers U01HG007480 and U54HG007480 to Christian Happi at Redeemer's

University, Ede, Osun State, Nigeria. This content is solely the responsibility of the authors and does not necessarily represent the official views of the National Institutes of Health. This project has also been supported by World Bank project ACE019 to Redeemer's University Nigeria (Christian Happi) and the Henry M Jackson Foundation to Redeemer's University Nigeria (Christian Happi).

\section{Availability of data and materials}

The dataset supporting the findings of this article is available from the corresponding author upon request.

\section{Ethics approval and consent to participate}

The study protocol was approved by The Ethics Committee of the Ministry of Health, Ibadan, Oyo State and National Health Research Ethics Committee, Abuja, Nigeria. Written informed consents were obtained from the parents/ guardians of the participating children.

\section{Consent for publication}

Not applicable.

\section{Competing interests}

The authors declare that they have no competing interests.

\section{Author details}

${ }^{1}$ Antimalarial Therapeutic Efficacy Monitoring Group, National Malaria Elimination Programme, The Federal Ministry of Health, Abuja, Nigeria. ${ }^{2}$ Department of Pharmacology and Therapeutics, University of Ibadan, Ibadan, Nigeria. ${ }^{3}$ Institute for Medical Research and Training, University of Ibadan, Ibadan, Nigeria. ${ }^{4}$ Department of Clinical Pharmacology, University College Hospital, Ibadan, Ibadan, Nigeria. ${ }^{5}$ Department of Biological Sciences and African Centre of Excellence for Genomics of Infectious Diseases (ACEGID), Redeemer University, Ede, Nigeria. ${ }^{6}$ Department of Paediatrics, University of Ibadan, Ibadan, Nigeria. ${ }^{7}$ Department of Medical Laboratory Science, University of Lagos, Lagos, Nigeria. ${ }^{8}$ Department of Pharmacology and Toxicology, University of Ibadan, Ibadan, Nigeria. ${ }^{9}$ Department of Paediatrics, University of Jos, Jos, Nigeria. ${ }^{10}$ Department of Pediatrics, Institute of Child Health, University of Nigeria Teaching Hospital, Enugu, Nigeria.

${ }^{11}$ Department of Paediatrics, University of Calabar, Calabar, Cross River State, Nigeria. ${ }^{12}$ Nigeria Institute of Medical Research, Lagos, Nigeria. ${ }^{13}$ Department of Paediatrics, Ahmadu Bello University, Zaria, Nigeria. ${ }^{14}$ Department of Paediatrics, Specialist Hospital, Maiduguri, Nigeria. ${ }^{15}$ Department of Paediatrics and Child Health, University of Ilorin, Ilorin, Nigeria. ${ }^{16}$ Department of Paediatrics, Federal Medical Centre, Yenagoa, Nigeria. ${ }^{17}$ Department of Paediatrics, Nnamdi Azikiwe University, Awka, Nigeria. ${ }^{18}$ Department of Paediatrics, Uthman Dan Fodio University, Sokoto, Nigeria. ${ }^{19}$ Department of Paediatrics, University of Maiduguri, Maiduguri, Nigeria. ${ }^{20}$ Department of 
Paediatrics, Imo State University Teaching Hospital, Orlu, Nigeria.

${ }^{21}$ Department of Medical Microbiology and Parasitology, University of Lagos, Lagos, Nigeria. ${ }^{22}$ Department of Medical Laboratory Science, University of Calabar, Calabar, Nigeria. ${ }^{23}$ Department of Biochemistry, Covenant University, Ota, Nigeria. ${ }^{24}$ World Health Organization, Country Office, Kampala, Uganda. ${ }^{25}$ Department of Physiology, University of Ibadan, Ibadan, Nigeria.

${ }^{26}$ University of Ibadan Research Foundation, University of Ibadan, Ibadan, Nigeria.

Received: 22 January 2019 Accepted: 9 July 2019

Published online: 06 August 2019

\section{References}

1. Noedl H, Se Y, Schaecher K, Smith BL, Socheat D, Fukuda MM. Evidence of artemisinin resistance in western Cambodia. New Engl J Med. 2008;359: 2619-20.

2. Noedl H, Se Y, Sriwichai S, Schaecher K, Teja-Isavadharm P, Smith B, et al. Artemisinin resistance in Cambodia: a clinical trial designed to address an emerging problem in Southeast Asia. Clin Infect Dis. 2010;51:e82-9.

3. Carrara VI, Zwang J, Ashley EA, Price RN, Stepniewka K, Marion B, et al. Changes in the treatment responses to artesunate-mefloquine on the northwestern border of Thailand during 13 years of continuous deployment. PLoS One. 2009;4:e4551.

4. Dondorp AM, Nosten F, Poravuth Y, Das D, Phyo AP, Tarning J, et al. Artemisinin resistance in Plasmodium falciparum. New Engl J Med. 2009;361: 455-67.

5. Phyo AP, Ashley EA, Anderson TJC, Bozdech Z, Carrara VI, Sriprawat K, et al. Declining efficacy of artemisinin combination therapy against $P$. falciparum malaria on the Thai-Myanmar border (2003-2013): the role of parasite genetic factors. Clin Infect Dis. 2016;63:784-91.

6. Phyo AP, Nkhoma S, Stepniewska K, Ashley EA, Nair S, McGready R, et al. Emergence of artemisinin-resistant malaria on the western border of Thailand: a longitudinal study. Lancet. 2012;379:1960-6.

7. Imwong M, Suwannasin K, Kunasol C, Sutawong K, Mayxay M, Rekol H, et al. The spread of artemisinin-resistant Plasmodium falciparum in the greater Mekong subregion: a molecular epidemiology observational study. Lancet Infect Dis. 2017;17:491-7. https://doi.org/10.1016/\$1473-3099(17)30048-8.

8. World Health Organization. Status Report on Artemisinin and Artemisininbased Combination Therapy Resistance. Geneva: WHO; 2017. https://www. who.int/malaria/publications/atoz/artemisinin-resistance-april2017/en/

9. Maiga AW, Fofana B, Sagara I, Dembele D, Dara A, Traore OB, et al. No evidence of delayed parasite clearance after oral artesunate treatment of uncomplicated falciparum malaria in Mali. Am J Trop Med Hyg. 2012;87:23-8.

10. Ménard D, Khim N, Begham J, Adegnika AA, Shafiul-Alam M, Amodu O, et al. For the KARMA consortium: a worldwide map of Plasmodium falciparum K13-propeller polymorphism. New Engl J Med. 2016;374:2453-64. https:// doi.org/10.1056/NEJMoa1513137.

11. Sagara I, Fofana B, Traore A, Diallo N, Diakite $H$, Togo AH, et al. Pyronaridine-artesunate or dihydroartemisinin-piperaquine versus current first-line therapies for repeated treatment of uncomplicated malaria: a randomised, multicentre, open-label, longitudinal, controlled, phase 3b/4 trial. Lancet. 2018:391:1378-90.

12. Borrmann S, Sasi P, Mwai L, Bashraheil M, Abdallah A, Muriithi S, et al. Declining responsiveness of Plasmodium falciparum infections to artemisinin-based combination treatments on the Kenyan coast. PLoS One. 2011;6:26005.

13. Bosman A, Mendis KN. A major transition in malaria treatment: the adoption and deployment of artemisinin-based combination therapies. Am J Trop Med Hyg. 2007;77(Suppl 6):193-7.

14. Federal Ministry of Health. National Antimalarial Treatment Guidelines. Abuja: Federal Ministry of Health; 2005.

15. Oguche S, Okafor HU, Watila I, Meremikwu M, Agomo P, Ogala W, et al. Efficacy of artemisinin-based combination treatments of uncomplicated falciparum malaria in under-five year-old Nigerian children. Am J Trop Med Hyg. 2014;91:925-35.

16. Ebenebe JC, Ntadom G, Ambe J, Wammanda R, Jiya N, Finomo F, et al. Efficacy of artemisinin-based combination treatments of uncomplicated falciparum malaria in under-five-year-old Nigerian children ten years after adoption as first-line antimalarials. Am J Trop Med Hyg. 2018:99:649-64. https://doi.org/10.4269/ajtmh.18-0115.
17. World Health Organization. Severe falciparum malaria. Trans R Soc Trop Med Hyg. 2000;94(Suppl. 1):1-90.

18. World Health Organization. Severe malaria. Trop Med Int Health. 2014; 19(Suppl. 1):7-131.

19. Sowunmi A, Akano K, Ayede Al, Ntadom G, Aderoyeje T, Adewoye EO, Fatunmbi B. Clinical illness and outcomes in Nigerian children with lateappearing anaemia after artemisinin-based combination treatments of uncomplicated falciparum malaria. BMC Infect Dis. 2016;16:240.

20. World Health Organization. Assessment and Monitoring of Antimalarial Drug. Efficacy for the treatment of uncomplicated falciparum malaria. Geneva: WHO/HTM/RBM/2003.50; 2003.

21. Sowunmi A, Akano K, Ntadom G, Ayede Al, Ibironke FO, Aderoyeje T, et al Therapeutic efficacy and effects of artemisinin-based combination treatments on uncomplicated Plasmodium falciparum malaria-associated anaemia in Nigerian children during seven years of adoption as first-line treatments. Infect Dis Poverty. 2017;6:36.

22. Sowunmi A, Akano K, Ayede Al, Adewoye EO, Ntadom G, Fatunmbi B, et al. Early rising asexual parasitaemia in Nigerian children following a first dose of artemisinin-based combination treatments of falciparum malaria. BMC Infect Dis. 2017;17:110

23. Happi CT, Gbotosho GO, Sowunmi A, Falade CO, Akinboye DO, Hudson T, et al. Molecular analysis of Plasmodium falciparum recrudescent malaria infections in children treated with chloroquine in Nigeria. Am J Trop Med Hyg. 2004;70:20-6.

24. Sowunmi A, Falade AG, Adedeji AA, Ayede Al, Fateye BA, Sowunmi CO, Oduola AMJ. Comparative Plasmodium falciparum kinetics during treatment with amodiaquine and chloroquine in children. Clin Drug Invest. 2001;21: 371-81.

25. Gbotosho GO, Sowunmi A, Happi CT, Okuboyejo TM. Therapeutic efficacies of artemisinin-based combination therapies in Nigerian children with uncomplicated falciparum malaria during five years of adoption as first-line treatments. Am J Trop Med Hyg. 2011;84:936-43.

26. Anon. Epi Info Version 7. A Word Processing Data Base and Statistics Program for Public Health on IBM-compatible Microcomputers. Atlanta: Centers for Disease Control and Prevention; 2016.

27. Anon. SPSS for Windows Release 22.0 (standard version). Armonk, New York: International Business Machine (IBM) Corporation; 2013.

28. White NJ. Antimalarial drug resistance. J Clin Invest. 2004;113:1084-92.

29. White NJ. Malaria parasite clearance. Malaria J. 2017;16:88.

30. Kyaw MP, Nyunt MH, Chit C, Aye MM, Aye KH, Aye MM, et al. Reduced susceptibility of Plasmodium falciparum to artesunate in southern Myanmar. PLoS One. 2013;8:e57689.

31. Outtara A, Kone A, Adams M, Fofana B, Maiga AW, Hampton S, et al. Polymorphisms in the K13-propeller gene in artemisinin-susceptible Plasmodium falciparum parasites from Bougoula-Hameau and Bandiagara, Mali. Am J Trop Med Hyg. 2015;92:1202-6.

32. Lu P, Cullerton R, Zhang M, Ramaprasad A, von Seidlein L, Zhou H, et al. Emergence of indigenous artemisinin-resistant Plasmodium falciparum in Africa. New Eng J Med. 2017;376:991-3.

33. White NJ. Assessment of the pharmacodynamics properties of antimalarial drugs in vivo. Antimicrob Agents Chemother. 1997;41:1413-22.

34. White NJ, Pongtavornpinyo W, Maude RJ, Saralamba S, Aguas R, Stepniewska K, et al. Hyperparasitaemia and low dosing are an important source of anti-malarial drug resistance. Malaria J. 2009:8:253.

35. Oduola AMJ, Sowunmi A, Milhous WK, Martin RK, Walker O, Salako LA Innate resistance to new antimalarial drugs in Plasmodium falciparum from Nigeria. Trans R Soc Trop Med Hyg. 1992;86:123-6.

36. Bustamante C, Folarin OA, Gbotosho GO, Batista CN, Mesquita EA, Brindeiro $\mathrm{RM}$, et al. In vitro reduced susceptibility to artemether in $P$. falciparum and its association with polymorphisms on transporter genes. J Infect Dis. 2012; 206:324-32 